\title{
Marantic Endocarditis
}

\section{Associated with Pancreatic Cancer: A Case Series}

\author{
Gayle S. Jameson ${ }^{\text {a }}$ Ramesh K. Ramanathan ${ }^{\text {a }}$ \\ Mitesh J. Borad ${ }^{b}$ Molly Downhour ${ }^{\mathrm{a}}$ Ronald Korn ${ }^{\mathrm{c}}$ \\ Daniel Von Hoff ${ }^{a}$
}

aScottsdale Clinical Research Institute, TGen Clinical Research Services,

${ }^{b}$ Mayo Clinic, and 'Scottsdale Medical Imaging, Scottsdale, Ariz., USA

\section{Key Words}

Marantic endocarditis - Pancreatic cancer - Nonbacterial thrombotic endocarditis

\begin{abstract}
Marantic endocarditis, otherwise known as nonbacterial thrombotic endocarditis (NBTE), is a well-documented phenomenon due to hypercoagulability from an underlying cause. It has been associated with a variety of inflammatory states including malignancy. Surprisingly, although hypercoagulability is often seen in patients with pancreatic cancer, marantic endocarditis has rarely been reported antemortem in this population. We report three cases of marantic endocarditis in patients with advanced pancreatic cancer. In two instances, the patients' neurological symptoms preceded the diagnosis of advanced pancreatic cancer. Health care professionals should be alert to the possibility of marantic endocarditis in any patient with cancer, especially pancreatic cancer, who presents with symptoms of neurological dysfunction or an arterial thrombotic event. Prompt diagnosis and treatment with heparin, unfractionated or low molecular weight, may prevent catastrophic CNS events and decrease morbidity in patients with pancreatic cancer and other malignancies.
\end{abstract}

\section{Case 1}

A 72-year-old male with stage II pancreatic cancer underwent a Whipple procedure. Nine months later, he experienced disease recurrence in the liver and was treated on a phase II clinical trial without disease response. The patient then developed leg edema and a venous Doppler study confirmed deep vein thrombus. Re-staging CT scans incidentally noted a pulmonary embolus. He was treated with low molecular weight heparin (LMWH). Two weeks later, the patient developed ataxia with repeated falls, and motor/sensory dysfunction of the right 4 th and 5th fingers. MRI of the brain confirmed multiple embolic infarctions throughout both cerebral hemispheres and cerebellum. Findings were consistent with a central source of emboli resulting in multiple infarcts. Figure 1 illustrates diffusion weighted MRI. A single axial slice from MRI demonstrates multiple hyperintense signal foci in the bilateral 
centrum semiovale. The distribution of the signal abnormalities is compatible with multiple embolic infarcts. Additional foci were noted elsewhere in the bilateral cerebral hemispheres and in the cerebellum (not shown). A transesophageal echocardiogram (TEE) showed no valvular vegetation or thrombi, but the quality of the study was limited. Based on the clinical picture and MRI results, the diagnosis of marantic endocarditis was made. The patient continued on LMWH and neurological symptoms worsened. Unfortunately, he continued to deteriorate from the pancreatic cancer and died 6 weeks after this hypercoagulable state was diagnosed.

\section{Case 2}

A 64-year-old male with a complex past medical history presented with recent onset of severe frontal headaches. Neurological work-up was unrevealing. Three months later, he experienced a seizure and temporary hemianopsia. He was found to have an occipital lobe infarct and was started on warfarin and phenytoin. One month later, he developed acute chest pain and was hospitalized. Cardiac enzymes were normal. A TEE noted vegetation on the anterior leaflet of the mitral valve. Workup for bacterial endocarditis was negative. The clinical picture was consistent with marantic endocarditis. The patient continued on warfarin. Additional studies revealed a second embolic stroke, a mass in the head of the pancreas, and multiple liver, lung and adrenal lesions. CA19-9 was 9,500 U/ml. Pathology of a lung mass showed mucinous adenocarcinoma consistent with a pancreas primary malignancy. The patient was treated with erlotinib and gemcitabine. He experienced a response to therapy by improvement in PET/CT and marked decrease in CA 19-9 to $156 \mathrm{U} / \mathrm{ml}$. During this time of tumor regression, he reported no episodes of seizures, severe headaches, visual changes or chest pain. However, re-staging after 6 months of therapy demonstrated radiologic and serologic evidence of disease progression and he was referred for a phase I trial. Unfortunately, due to onset of pneumonia, treatment on a clinical trial was not initiated. He had recurrence of seizures and MRI of the brain noted interval development of sub-acute infarcts in the left occipital lobe and ischemic changes in the left parietal lobe. Anticoagulation was changed to enoxaparin. The patient was not able to receive additional anti-tumor therapy due to persistent infection and worsening performance status. He died 2 months after ceasing chemotherapy, 9 months after initial diagnosis.

\section{Case 3}

A 61-year-old male with no significant past medical history underwent a left foot bunionectomy. One week later, he developed bilateral calf pain and leg edema. Then he experienced a syncopal event and was transported to a local emergency department with complaint of pleuritic chest pain. He also reported a 2-3 month history of fatigue and weight loss. CT scan of the brain showed no evidence of mass, hemorrhage or infarction. A chest CT angiogram revealed multiple bilateral pulmonary emboli. A TEE suggested the presence of a string thrombus in transit across the intra-atrial septum. Venous Doppler studies of the lower extremities noted multiple bilateral, sub-acute non-occluding thrombi. The patient was anticoagulated with LMWH followed by warfarin. Further workup revealed a $3 \times 3 \times 6 \mathrm{~cm}$ mass in the tail of the pancreas with multiple metastatic lesions in the liver and lungs. Serum CA 19-9 level was 40,606 U/ml. Liver biopsy revealed a moderately well differentiated adenocarcinoma consistent with pancreas as the primary site of malignancy. Three weeks after diagnosis of stage IV pancreatic cancer, pulmonary emboli, and Trousseau's syndrome, the patient began treatment on a gemcitabine based phase I clinical trial. Warfarin was stopped and the patient continued daily enoxaparin. During the first week of chemotherapy, on three separate occasions, he experienced a transient loss of visual fields of the right eye, lasting less than one minute. Ophthalmologic exam revealed no fundoscopic abnormalities. Repeat CT scan of the brain again showed no abnormalities. Carotid Doppler studies were negative. Thus, the patient's repeated temporary vision loss was attributed to embolic phenomenon with transient ischemic attacks. Enoxaparin was changed to twice daily dosing. The TEE was repeated, now noting vegetations on the mitral valve. As noted in figure 2 , a four chamber echocardiogram demonstrates thickened echogenic leaflets of the mitral valve (white arrows) compatible with vegetations. Notice the thin appearance of the leaflets on the tricuspid valve for comparison (arrowheads). Further evaluation for bacterial endocarditis was negative, including multiple sterile blood cultures. The diagnosis of marantic endocarditis was established. After six months of receiving the aforementioned chemotherapy on a clinical trial, restaging PET/CT scan shows response to chemotherapy with marked decrease $(50 \%)$ in SUV in pulmonary, hepatic and nodal metastases. Of note, the patient has had no recurrence of the visual abnormalities and no new neurological symptoms. He continues to self administer enoxaparin twice daily. He is feeling well and working full-time. 


\begin{tabular}{r|l|l|l} 
Case Reports $h$ & $\begin{array}{l}\text { Case Rep Gastroenterol 2009;3:67-71 } \\
\text { D01: 10.1159/000207195 }\end{array}$ & Published online: April 15, 2009 & $\begin{array}{l}\text { O 2009 S. Karger AG, Basel } \\
\text { ISSN 1662-0631 } \\
\text { www.karger.com/crg }\end{array}$ \\
\hline
\end{tabular}

\section{Discussion}

NBTE is a well-documented phenomenon due to hypercoagulability from an underlying cause. It has been associated with a variety of inflammatory states including malignancy. It is the result of a prothrombotic condition characterized by deposition of sterile fibrin and platelet-rich thrombi on previously undamaged heart valves. Cardiac function is generally unaffected. Clinical presentation is most often a sudden neurological deficit. Associated morbidity and mortality are due to increased frequency of arterial embolic events, specifically in the central nervous system [1]. The preferred diagnostic test for NBTE is a TEE, which is more sensitive in the detection of valvular vegetations than the transthoracic approach [2]. Recommended treatment is therapeutic anticoagulation with unfractionated heparin or LMWH and treatment of the underlying cause $[1,3]$. However, there have been no studies defining the optimal treatment of NBTE. Also, the benefit of heparin compared to warfarin in the treatment of NBTE has not been studied.

A review of the literature from the past 3 decades reveals multiple case reports associating marantic endocarditis with various malignancies, most commonly of gynecologic origin [4-8]. Surprisingly, although hypercoagulability is most often seen in patients with pancreatic cancer, marantic endocarditis has rarely been reported antemortem in this population [9-11]. A prospective echocardiographic study of 200 living patients with solid tumors demonstrated a high prevalence of cardiac valvular vegetations with an overall incidence of 19\%, including 3 of 6 pancreatic cancer patients (50\%) [12]. Recently, Smeglin et al. documented the first case of an antemortem diagnosis of marantic endocarditis as the initial presentation of an underlying pancreatic cancer [13].

Here we report three cases of marantic endocarditis in patients with advanced pancreatic cancer. In the latter two patients, neurological symptoms due to marantic endocarditis preceded the diagnosis of the pancreatic cancer. We suspect that given the incidence of hypercoagulability in patients with cancer of the pancreas, and that we have seen 3 cases in the past 18 months, marantic endocarditis is likely underreported and underdiagnosed. Of interest, we have also observed resolution of neurological symptoms in two patients, during the period of disease response to anti-tumor therapy while continuing on LMWH. This observation suggests that prompt recognition and treatment of marantic endocarditis along with effective treatment of the underlying malignancy may improve the dismal prognosis of this syndrome.

Health care professionals should be alert to the possibility of marantic endocarditis in any patient with cancer, especially pancreatic cancer, who presents with symptoms of neurological dysfunction or an arterial thrombotic event. In our experience, prompt diagnosis and treatment with heparin and treatment of the patient's pancreatic cancer may prevent catastrophic CNS events and decrease morbidity. 


\begin{tabular}{r|l|l|l} 
Case Reports $/$ h & $\begin{array}{l}\text { Case Rep Gastroenterol 2009;3:67-71 } \\
\text { D0I: 10.1159/000207195 }\end{array}$ & Published online: April 15, 2009 & $\begin{array}{l}\text { O 2009 S. Karger AG, Basel } \\
\text { ISSN 1662-0631 } \\
\text { www.karger.com/crg }\end{array}$ \\
\hline
\end{tabular}

Fig. 1. Diffusion weighted MRI. Single axial slice from MRI demonstrates multiple hyperintense signal foci in the bilateral centrum semiovale. The distribution of signal abnormality is compatible with multiple embolic infarcts. Additional foci were noted elsewhere in the bilateral cerebral hemispheres and in the cerebellum (not shown). The MRI was obtained on GE I.5 T with head coil, ET 1; TR $8000.0 \mathrm{~ms}$; TE $95.9 \mathrm{~ms}$; slice thickness $5.0 \mathrm{~mm} / 0.0 \mathrm{~mm}$ skip.

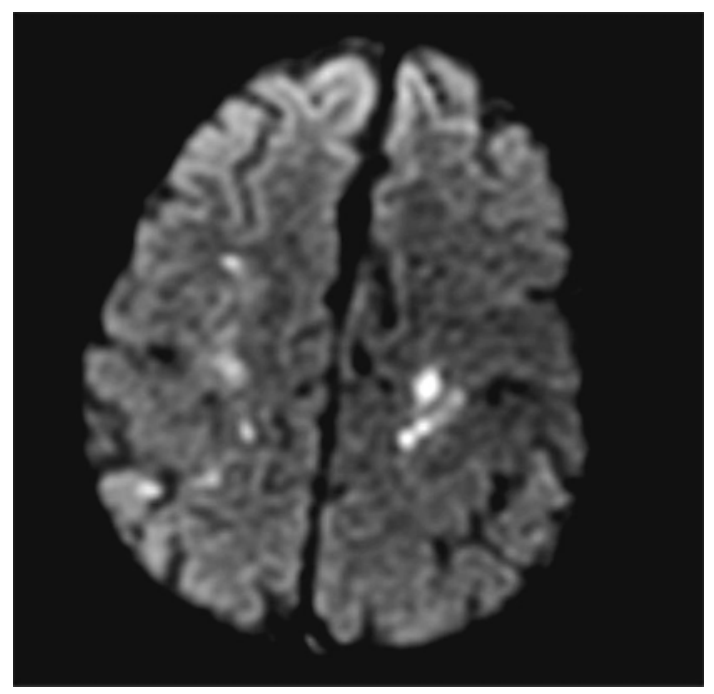

Fig. 2. Four chamber echocardiographic view demonstrating thickened echogenic leaflets of the mitral valve (arrows) compatible with vegetation. Notice the thin appearance of the leaflets on the tricuspid valve for comparison (arrowheads). $\mathrm{RA}=$ Right atrium; $\mathrm{LA}=$ left atrium; $\mathrm{RV}=$ right ventricle; $\mathrm{LV}=$ left ventricle.

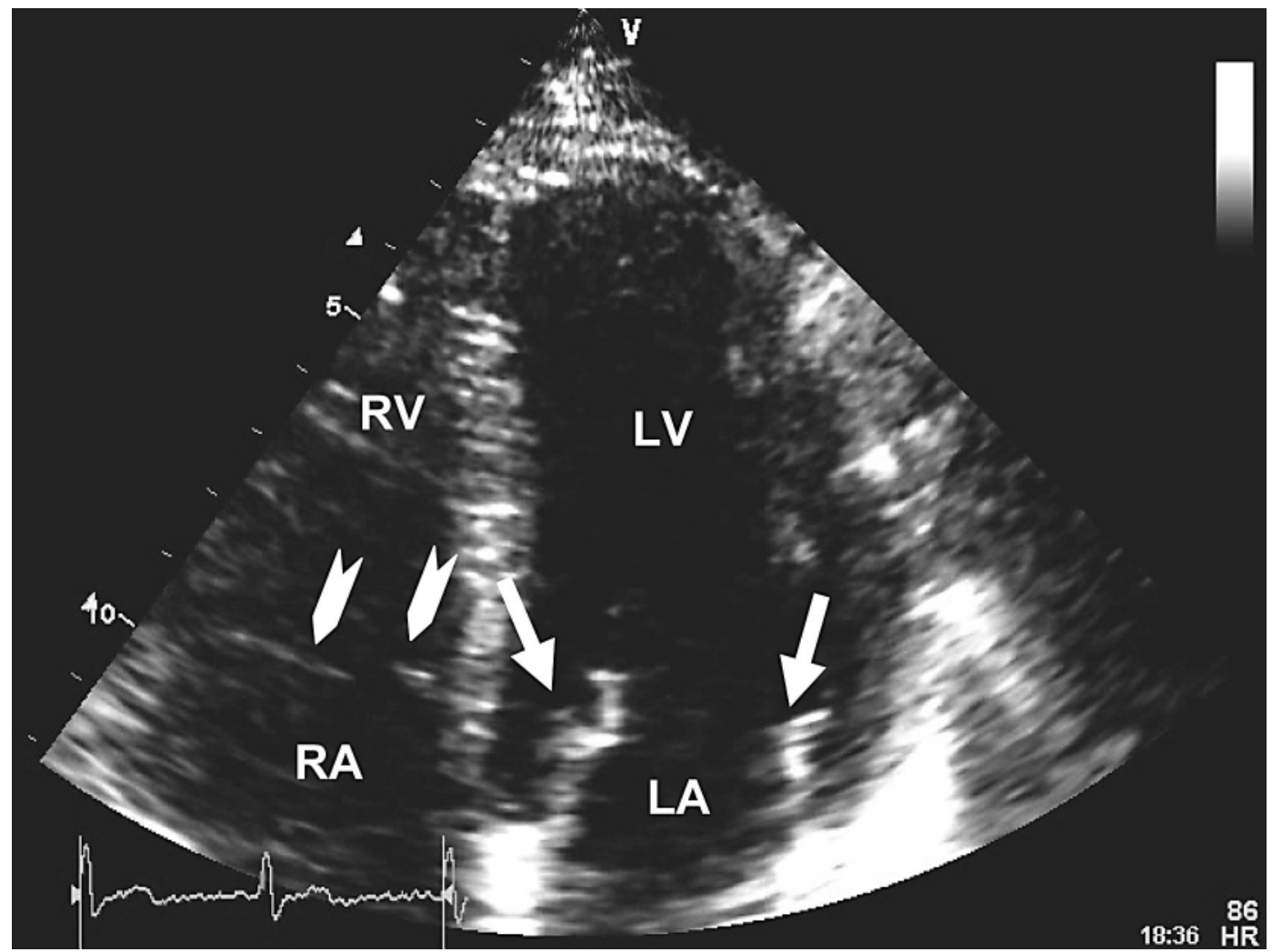




\section{References}

1 El-Shami K, Griffiths E, Streiff M: Nonbacterial thrombotic endocarditis in cancer patients: pathogenesis, diagnosis and treatment. Oncologist 2007;12:518-523.

2 Dutta T, Karas MG, Segal AZ, Kizer JR: Yield of transesophageal echocardiography for nonbacterial thrombotic endocarditis and other cardiac sources of embolism in cancer patients with cerebral ischemia. Am J Cardiol 2006;97:894-898

3 Salem DN, Stein PD, Al-Ahmad A, et al: Antithrombotic therapy in valvular heart disease - native and prosthetic: The Seventh ACCP Conference on Antithrombotic and Thrombolytic Therapy. Chest 2004;126(3 suppl):457S-482S.

4 Borowski A, Ghodsizad A, Cohnen M, Gams E: Recurrent embolism in the course of marantic endocarditis. Ann Thorac Surg 2005;79:2145-2147.

5 Numnum TM, Leath CA, Straughn MJ: Synchronous primary endometrial and ovarian carcinoma in a patient with marantic endocarditis. Obstetr Gynecol 2006;108(3 Pt 2):748-750.

-6 Glass JP: The diagnosis and treatment of stroke in a patient with cancer: nonbacterial thrombotic endocarditis (NBTE): a case report and review. Clin Neurol Neurosurg 1993;95:315-318.

7 Aryana A, Esterbrooks DJ, Morris PC: Nonbacterial thrombotic endocarditis with recurrent embolic events as manifestation of ovarian neoplasm. J Gen Intern Med 2006;21:C12-C15.

$\checkmark 8$ Borowski A, Ghodsizad A, Gams E: Stroke as a first manifestation of ovarian cancer. J Neurooncol 2005;71:267-269.

-9 Chen L, Li Y, Gebre W, Lin J: Myocardial and cerebral infarction due to nonbacterial thrombotic endocarditis as an initial presentation of pancreatic adenocarcinoma. Arch Pathol Lab Med 2004;128:1307-1308.

10 Rogers LR, Cho ES, Kempin S, Posner JB: Cerebral infaction from non-bacterial thrombotic endocarditis: clinical and pathological study including the effects of anticoagulation. Am J Med 1987;83:746-756.

11 Schattner A, Klepfish A, Huszar M, Shani A: Two patients with arterial thromboembolism among 311 patients with adenocarcinoma of the pancreas. Am J Med Sci 2002;24:335-338.

12 Edoute Y, Haim N, Rinkevich D, Brenner B, Reisner S: Cardiac valvular vegetations in cancer patients: a prospective echocardiographic study of 200 patients. Am J Med 1997;102:252-258.

13 Smeglin A, Ansari M, Skali H, Oo TH, Maysky M: Marantic endocarditis and disseminated intravascular coagulation with systemic emboli in presentation of pancreatic cancer. J Clin Oncol 2008;26:1383-1385. 